\title{
In-Situ S/TEM Probing of the Behavior of Nanoparticles Under Chemical and Electrochemical Reactions in the System Involving Solid, Liquid and Gas
}

\author{
Chongmin Wang ${ }^{1}$, Langli Luo ${ }^{1}$ and Yuyan Shao ${ }^{1}$ \\ 1. Pacific Northwest National Laboratory, Richland, WA.
}

Nanoparticles of either as an active functioning component or as a reaction product is of great importance for a range of science and engineering, such as catalysis, nucleation and growth, metal air-battery, and oxidation and corrosion. A typical example is the deactivation of catalyst particles. Deactivation of supported metal nanoparticle catalysts, especially in relevant gas condition, is a critical challenge for many technological applications, including heterogeneous catalysis, electrocatalysis and fuel cells. It has been commonly realized that deactivation of catalysts stems from surface area loss due to particle coarsening, however, for which the mechanism remains largely unclear. Direct in-situ TEM observation reveals unprecedented details at atomic scale with respect to the behavior of Pt nanoparticles for fuel cell application, especially the coarsening dynamics of Pt nanoparticles on bare CNTs surface and in Nafion covered $\mathrm{Pt} / \mathrm{CNT}$ s under $\mathrm{O}_{2}$ and $\mathrm{H}_{2} \mathrm{O}$ gas environment. We found that the coarsening of Pt nanoparticles proceeded through migration and coalescence process. The migration rates of $\mathrm{Pt}$ nanoparticles on bare CNT surface is much faster in $\mathrm{O}_{2}$ than that in $\mathrm{H}_{2} \mathrm{O}$. The strong oxygen chemisorption on Pt nanoparticles weakens the interaction between Pt and CNT surface, leading to a fast migration in $\mathrm{O}_{2}$. Incorporation of Nafion electrolyte layer put on a mechanically confinement to Pt/CNTs which reduces the Pt migration rate in $\mathrm{O}_{2}$. However, this mechanical confinement is largely relieved by introducing $\mathrm{H}_{2} \mathrm{O}$, and a lubricated interface is created, both leading to a faster migration rate of $\mathrm{Pt}$ in $\mathrm{H}_{2} \mathrm{O}$ than that in $\mathrm{O}_{2}$. These results bridge the gap between the previous in situ TEM studies in idealized conditions and the technological relevant conditions of $\mathrm{Pt}$ catalyst for PEMFC, thus provide insights to rational design of $\mathrm{Pt} /$ carbon catalysts incorporating Nafion electrolyte to achieve a long-lasting PEM fuel cells.

The Environmental TEM (FEI Titan ${ }^{\circledR}$ ETEM) is equipped with an objective-lens aberration corrector enabling sub-Angstrom HRTEM imaging. A customized gas delivery system enabling precise pressure control from the base pressure $\sim 2 \times 10^{-8}$ mbar to $10 \mathrm{mbar}$. A customized water vapor delivery system can introduce $\mathrm{H}_{2} \mathrm{O}$ gas into the ETEM with a purity of $>96 \%$ and up to a pressure of $0.1 \mathrm{mbar}$. The system is composed of a diaphragm pump, a vacuum gauge and a water reservoir. The diaphragm pump creates a vacuum to let the water vapor fill in the pipeline, and by repeating pumping-filling process, a high purity of water vapor can be achieved. The Pt/CNTs or Nafion/Pt/CNTs/ loaded on TEM grids were mounted on a Gatan ${ }^{\circledR}$ double-tilt heating holder (Model 652) and inserted into the TEM column. The sample was first brought up to $100{ }^{\circ} \mathrm{C}$ and then quickly exposed to the ambient gas of $\mathrm{O}_{2}, \mathrm{H}_{2}$ and $\mathrm{H}_{2} \mathrm{O}$ with a partial pressure $\sim 0.01 \mathrm{mbar}$. Based on our calibration of the effect of electron beam, we chose a beam dose rate of 300 electron $/ \AA^{2}$ s and take one image every 5 or 10 minutes for a total time span of 1 to 4 hours, leading to a typical cumulative beam dose of $7.2 \times 10^{3}$ to $1.44 \times 10^{4}$ electron $/ \AA^{2}$ with $1 \mathrm{~s}$ exposure for each image, which is far below the limit of the safe zone.

We revealed the atomistic process of the particle coalescence through in situ HRTEM imaging of two adjacent $\mathrm{Pt}$ nanoparticles and illustrated the promotion effect of $\mathrm{O}_{2}$ for particle coalescence by in-situ switching the gas environment from $\mathrm{H}_{2} \mathrm{O}$ to $\mathrm{O}_{2}$ while observing the dynamic behavior of the same pair of particles. To minimize the beam effect, beam-blank experiment was employed so that the sample was only exposed to beam for capturing the HRTEM images as shown in Figure 3. Two adjacent Pt nanoparticles 
with different surface facets were shown in close distance but separated in Figure 3 a imaging in vacuum at $100{ }^{\circ} \mathrm{C}$. After $\sim 20$ minutes in $\mathrm{H}_{2} \mathrm{O}$, the distance between these two particles slightly decreased, but they still maintained their surface facets as evidenced in Figure $1 \mathrm{~b}$. After switching the gas from $\mathrm{H}_{2} \mathrm{O}$ to $\mathrm{O}_{2}$, these two particles coalesced within two minutes to form a new particle with newly reconstructed surface facets as shown in Figure 1c. Sequential images clearly revealed that the first step for coalescence is the rotation of the particle at the left side as seen from the $0 \mathrm{~s}$ image, followed by the merging of the gap between these two nanoparticles as seen in images of $56 \mathrm{~s}$, and finally the formation of one larger single $\mathrm{Pt}$ NP as shown in the image of $118 \mathrm{~s}$. The newly formed Pt NP adopted the same orientation of the Pt NP at the right side, and the Pt NP at the left side went through a rotation of $\sim 85^{\circ}$ as illustrated in Figure 1d [2].

References:

[1] Yuyan Shao et al, Applied Catalysis B: Environmental. 79 (2008), p. 89.

[2] The work was conducted in the William R. Wiley Environmental Molecular Sciences Laboratory (EMSL), a national scientific user facility sponsored by DOE's Office of Biological and Environmental Research and located at PNNL.

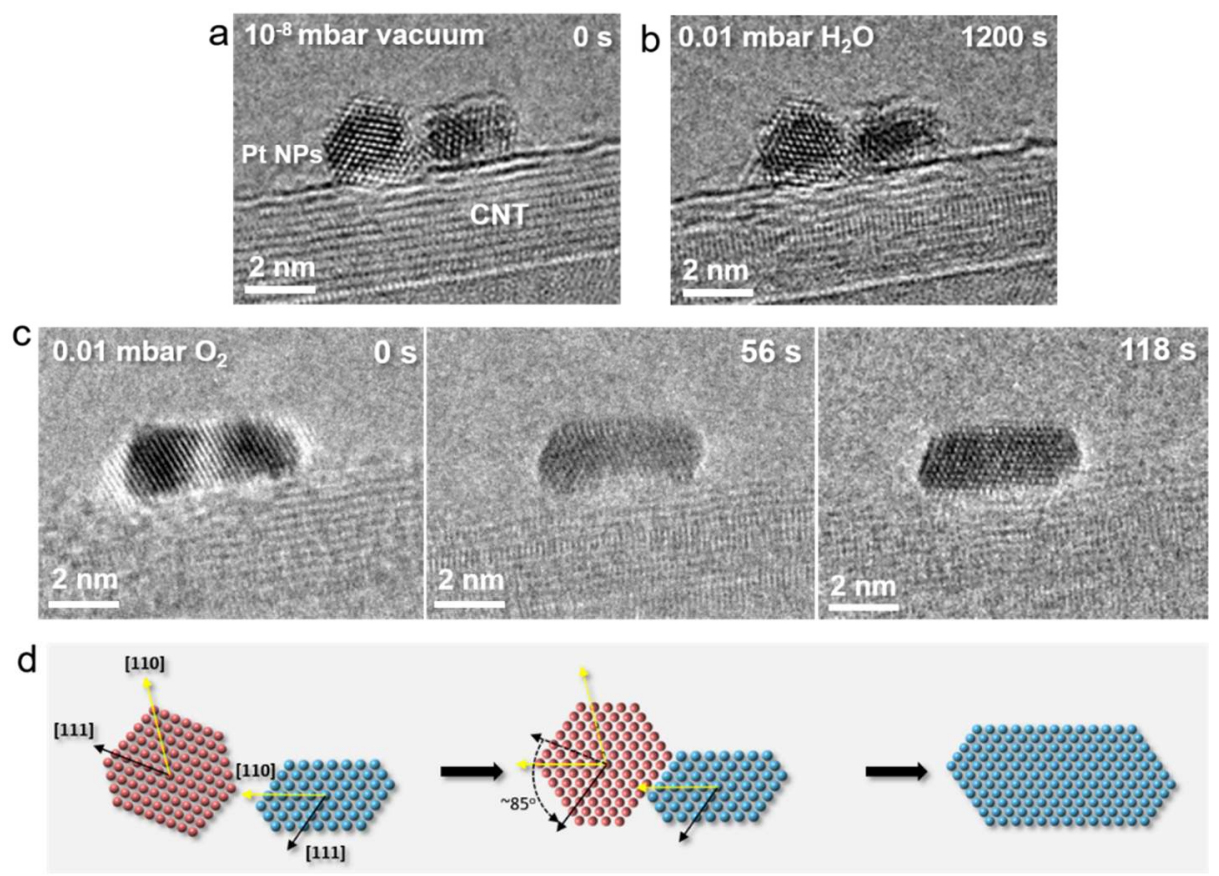

Figure 1. Migration and coalescence process of Pt/CNTs at $100{ }^{\circ} \mathrm{C}$. (a) HRTEM image shows two separated Pt nanoparticles in vacuum. (b) HRTEM image shows the distance between two Pt nanoparticles decreased but still maintained their individual shape and orientation after 30 minutes in $\mathrm{H}_{2} \mathrm{O}$. (c) A series of time-lapsed HRTEM images show the coalescence process of two Pt nanoparticles after switching gas from $\mathrm{H}_{2} \mathrm{O}$ to $\mathrm{O}_{2}$. (d) Schematic illustrates the coalescence of these two Pt nanoparticles involves rotation of one NP and growth through orientation match. 